\title{
The effect of Omega-3 polyunsaturated fatty acid supplementation on exercise- induced muscle damage
}

\author{
Yvoni Kyriakidou', Carly Wood ${ }^{1,2}$, Chrystalla Ferrier ${ }^{1}$, Alberto Dolci ${ }^{1+}$ and Bradley Elliott ${ }^{1 *+}$ (D)
}

\begin{abstract}
Background: Exercise-induced muscle damage (EIMD) results in transient muscle inflammation, strength loss, muscle soreness and may cause subsequent exercise avoidance. Omega-3 (n-3) supplementation may minimise EIMD via its anti-inflammatory properties, however, its efficacy remains unclear.

Methods: Healthy males ( $n=14,25.07 \pm 4.05$ years) were randomised to $3 \mathrm{~g} /$ day $n-3$ supplementation $(\mathrm{N}-3, n=7)$ or placebo (PLA, $n=7$ ). Following 4 weeks supplementation, a downhill running protocol (60 min, $65 \% \mathrm{~V}_{2} \mathrm{max}_{1}-10 \%$ gradient) was performed. Creatine kinase (CK), interleukin (IL)-6 and tumour necrosis factor (TNF)-a, perceived muscle soreness, maximal voluntary isometric contraction (MVIC) and peak power were quantified pre, post, and 24, 48 and $72 \mathrm{~h}$ post-EIMD.

Results: Muscle soreness was significantly lower in N-3 vs PLA group at $24 \mathrm{~h}$ post-EIMD $(p=0.034)$. IL-6 was increased in PLA ( $p=0.009)$ but not in N-3 ( $p=0.434)$ following EIMD, however, no significant differences were noted between groups. Peak power was significantly suppressed in PLA relative to pre-EIMD but not in N-3 group at $24 \mathrm{~h}$ post-EIMD. However, no significant difference in peak power output was observed between groups. MVIC, CK and TNF-a were altered by EIMD but did not differ between groups.

Conclusion: $\mathrm{N}-3$ supplementation for 4 weeks may successfully attenuate minor aspects of EIMD. Whilst not improving performance, these findings may have relevance to soreness-associated exercise avoidance.

Keywords: Omega-3, Supplementation, Ergogenic aid, Eccentric exercise, Muscle damage, EIMD, DOMS, Recovery, Inflammation, Performance
\end{abstract}

\section{Background}

The recovery from vigorous athletic performance concerns many groups of people, from high performance athletes to recreationally active individuals. Eccentric exercise, especially novel or high-force eccentric protocols, can produce substantial muscle fibre damage $[1,2]$. Such vigorous-intensity exercise may lead to exercise-induced muscle damage (EIMD) [3]. Symptoms of EIMD include

\footnotetext{
* Correspondence: b.elliott@westminster.ac.uk

${ }^{\dagger}$ Alberto Dolci and Bradley Elliott are joint senior authors.

${ }^{1}$ Translational Physiology Research Group, School of Life Sciences, College of Liberal Arts and Sciences, University of Westminster, London W1W 6UW, UK Full list of author information is available at the end of the article
}

pain, swelling, muscle strength and power loss, reduced range of motion (ROM), delayed onset muscle soreness (DOMS) and impaired recovery $[4,5]$; resulting in impairment of exercise performance [6]. Functionally, muscle strength is reduced by $\sim 20$ to $50 \%$ immediately and post-exercise can take between 2 to 7 days to fully recover [7]. Systemically, EIMD is paralleled by an inflammatory response involving many mediators, such as interleukin -1 receptor antagonist (IL-1ra), interleukin (IL)-6, IL-10 and acute phase proteins [8], and a release of muscle specific creatine kinase $[1,9]$. Strategies to reduce muscle damage and inflammation following EIMD

C C The Author(s). 2021 Open Access This article is licensed under a Creative Commons Attribution 4.0 International License, which permits use, sharing, adaptation, distribution and reproduction in any medium or format, as long as you give appropriate credit to the original author(s) and the source, provide a link to the Creative Commons licence, and indicate if changes were made. The images or other third party material in this article are included in the article's Creative Commons licence, unless indicated otherwise in a credit line to the material. If material is not included in the article's Creative Commons licence and your intended use is not permitted by statutory regulation or exceeds the permitted use, you will need to obtain permission directly from the copyright holder. To view a copy of this licence, visit http://creativecommons.org/licenses/by/4.0/ The Creative Commons Public Domain Dedication waiver (http://creativecommons.org/publicdomain/zero/1.0/) applies to the data made available in this article, unless otherwise stated in a credit line to the data. 
can therefore be of use to individuals interested in increasing their rate of recovery and maintaining performance.

Omega-3 polyunsaturated fatty acids ( $n-3$ PUFA) have a double bond that is closest to the methyl terminus $\left(-\mathrm{CH}_{3}\right)$ of the acyl chain [10]. N-3 PUFA are incorporated into phospholipids, altering cell membranes, which typically contain a high proportion of arachidonic acid (AA). This results in increased accumulation of eicosapentaenoic acid (EPA) and docosahexaenoic acid (DHA) and at the parallel decline of AA; and potentially blunting reactive oxygen species (ROS) and inflammatory cytokine production [10]. Anti-inflammatory mediators derived from n-3 PUFA and its main bioactive fatty acids, such as EPA and DHA, have been recognised along with their mechanism of their action [10]. It has been suggested that $n-3$ PUFA may prove a viable strategy to attenuate muscle inflammation and improve functional recovery following high-intensity exercise [11]. One of the connections between n-3 PUFA and muscle inflammation is via down-regulation of proinflammatory cytokines, such as TNF- $\alpha$ and IL-6, reduced production of AA and ROS, consequently, resulting in a decrease in the inflammatory response [12].

Increasing evidence suggests that $\mathrm{n}-3$ supplementation impairs pro-inflammatory cytokines and ROS production, and hence may show a direct relationship between intense exercise recovery and markers of inflammation $[12,13]$. Although animal studies have shown mixed results when evaluating the efficacy of $\mathrm{n}-3$ supplementation on muscle damage, exercise metabolism and exercise performance; human studies have demonstrated that physiological parameters that are linked to improved physical performance and oxygen utilisation, such as blood flow during exercise, can be augmented by dietary n-3 PUFA [11, 14]. Tarbinian et al. [15], Jouris et al. [16] and Jakeman et al. [17] have shown a pain reduction following EIMD with n-3 supplementation. A recent meta-analysis [18] also concluded that $n-3$ supplementation could alleviate DOMS after eccentric exercise. Additionally, Atashak et al. [19] reported substantial reduction in $\mathrm{CK}$ and in C-reactive protein after lower body resistance exercise following 1 week of $540 \mathrm{mg}$ EPA and $360 \mathrm{mg}$ DHA. Further, other studies $[20,21]$ have demonstrated that $n-3$ supplementation has a positive effect on eccentric exercise protocols by reducing the concentrations of IL- 6 and TNF- $\alpha$. However, mixed results have been reported to date, with others $[22,23]$ observing no effect of $n-3$ supplementation on exercise-induced inflammatory and muscle damage markers, and functional markers, such as maximal voluntary contraction (MVC) and DOMS.

It remains unclear whether $\mathrm{n}-3$ supplementation has any beneficial effect in blunting the effects of EIMD, either by increasing the rate of recovery of functional performance, by reducing circulating pro-inflammatory cytokines, or both. Due to this lack of clarity in the literature, the aim of the current study was to add evidence by assessing the effect of $\mathrm{n}-3$ supplementation on EIMD following a downhill running bout. It was hypothesised that $3 \mathrm{~g}$ of $\mathrm{n}-3$ supplementation for 4 weeks would attenuate muscle inflammation following EIMD which subsequently would decrease the recovery time, and thus improve exercise performance.

\section{Methods \\ Participants}

Ethical approval was obtained by the College of Liberal of Arts and Sciences Research Ethics Committee, University of Westminster (ETH1617-0182). All work herein conforms to the standards set by the Declaration of Helsinki of 1975. Written informed consent was obtained from all participants prior to their participation.

A total of 23 healthy, physically active males (self-reported: 4-5 times weekly structured exercise) aged 1835 years of age were recruited to participate in this experimental study. Following withdrawals $(n=9$; inability to attend all visits, injury or illness outside of trials or inability to complete downhill protocol), 14 participants ( $25.07 \pm 4.05$ years of age) completed the protocol and are included in the analysis below.

Participants were required to refrain from any structured exercise for $48 \mathrm{~h}$, and from alcohol and caffeine $24 \mathrm{~h}$ prior to baseline visit and EIMD protocol. They were also asked to refrain from eccentric strenuous exercise during the 5 weeks of the study as well as in the following $72 \mathrm{~h}$ after the muscle-damaging exercise bout. Exclusion criteria included age (outside 18-35 age range), smoking, sex, taking any medication (e.g. nonsteroidal anti-inflammatory drugs), and consuming fish oil supplements $<6$ months prior to commencing the study and the presence of any known immune, cardiovascular or metabolic disease. To further confirm participants were free from upper respiratory tract infections, they completed an illness-specific questionnaire (WURSS-21) [24]. Additionally, participants were free from any pain or injury as determined by the Physical Activity Readiness Questionnaire (PAR-Q) pre-exercise participation screening. Participants were also excluded if they regularly undertook downhill running or eccentric exercise (e.g. resistance exercise, squats) as part of their normal training $<6$ months prior to commencing the study.

\section{Experimental design}

All participants were required to attend the human performance laboratory in the morning on 5 occasions. During visit 1 , in an overnight fasted-state, participants 
performed baseline measurements which included anthropometric measurements, a urine sample and a venous blood sample. Perceived muscle soreness, maximal voluntary isometric contraction (MVIC) on the leg and anaerobic peak power via Wingate test were determined as indirect markers of muscle damage, described fully below.

Following baseline measurements, participants performed a treadmill $\dot{\mathrm{V}}_{2}$ max test (HP Cosmos Mercury 4.0, Nussdorf-Traunstein, Germany) with expired gases analysed by an on-line breath-by breath system (Cortex Metalyser 3B, Biophysik, Leipzig, Germany). The Metalyser was calibrated according to manufacturer's guidelines prior to each test. Following completion of the $\dot{\mathrm{VO}}_{2}$ max test, a 6-min running speed $\left(\mathrm{V}_{\text {test }}\right)$ at $65 \%$ $\dot{\mathrm{V}}_{2}$ max verification was performed on a downhill run at $-10 \%$ gradient, as we have previously reported [25]. After baseline testing, participants were single-blind randomised to either N-3 ( $3 \mathrm{~g} /$ day of $\mathrm{n}-3$ PUFA) or PLA (placebo) group by a computer-generated block randomization in advance (http://www.randomization. com).

Two weeks before beginning testing, participants filled a health questionnaire (WURSS-21) on each of the 14 days preceding trial to ensure that they were free from common cold symptoms before testing. In visit 2, participants reported to the laboratory at 07:00 am having fasted overnight and performed the EIMD protocol (downhill running; 60 min at $65 \% \dot{\mathrm{VO}}_{2}$ max with a $-10 \%$ gradient). All above measurements were repeated prior to- and immediately-post the EIMD trial. One day before the visit participants were asked to consume water based on their body mass $(5 \mathrm{~mL} / \mathrm{kg})$ [25] before they reported to the laboratory to ensure adequate hydration before exercise. Identical follow up assessments, except urine sample, were repeated at visits 3-to-5 (24, 48 and $72 \mathrm{~h}$ post-EIMD), during which participants were in a non-fasted state. An overview of the study design is presented in Fig. 1.

\section{Hydration status}

Urine sample was collected at baseline and pre-EIMD to assess participants' hydration status. Hydration was verified by checking that urine specific gravity (USG) (Atago MASTER-SUR/Na refractometer, Atago Co., Ltd. Tokyo, Japan) upon arrival was between 1.001 and 1.029 [26]. Urine colour was also checked by using the validated urine colour chart (1-8 scale) [26].

\section{Anthropometric measurements}

Height (to nearest $0.1 \mathrm{~cm}$ ) was measured using a wallmounted "Harpenden" stadiometer (Holtain Ltd., Crymych, Wales, UK) fitted with a high speed Veeder-Root counter (Veeder-Root, Elizabethtown, NC, USA) with participants stood in bare feet, heels together with their shoulders and buttocks in contact with the stadiometer. Body weight (to nearest $0.1 \mathrm{~kg}$ ), BMI and body fat \% (to nearest $0.1 \%$ ) were measured using BIA (Tanita SC330ST, Tokyo, Japan) with participant being fasted and with an empty bladder.

\section{Supplementation}

Omega-3 supplementation consisted of 3 gelatine-coated capsules per day ( 1 consumed in the morning, 1 at lunch and 1 in the evening), each containing $1040 \mathrm{mg}$ of $\mathrm{n}-3$ PUFA (715 mg of EPA and $286 \mathrm{mg}$ DHA) per capsule (Maximum Strength Pure Fish Oil, Nature's Best, UK) for a total of $3900 \mathrm{mg}$ of fish oil daily, containing $3 \mathrm{~g}$ of n-3 PUFA (2145 mg of EPA and $858 \mathrm{mg}$ DHA) per day for a period of 4 weeks. Whilst commonly reported side

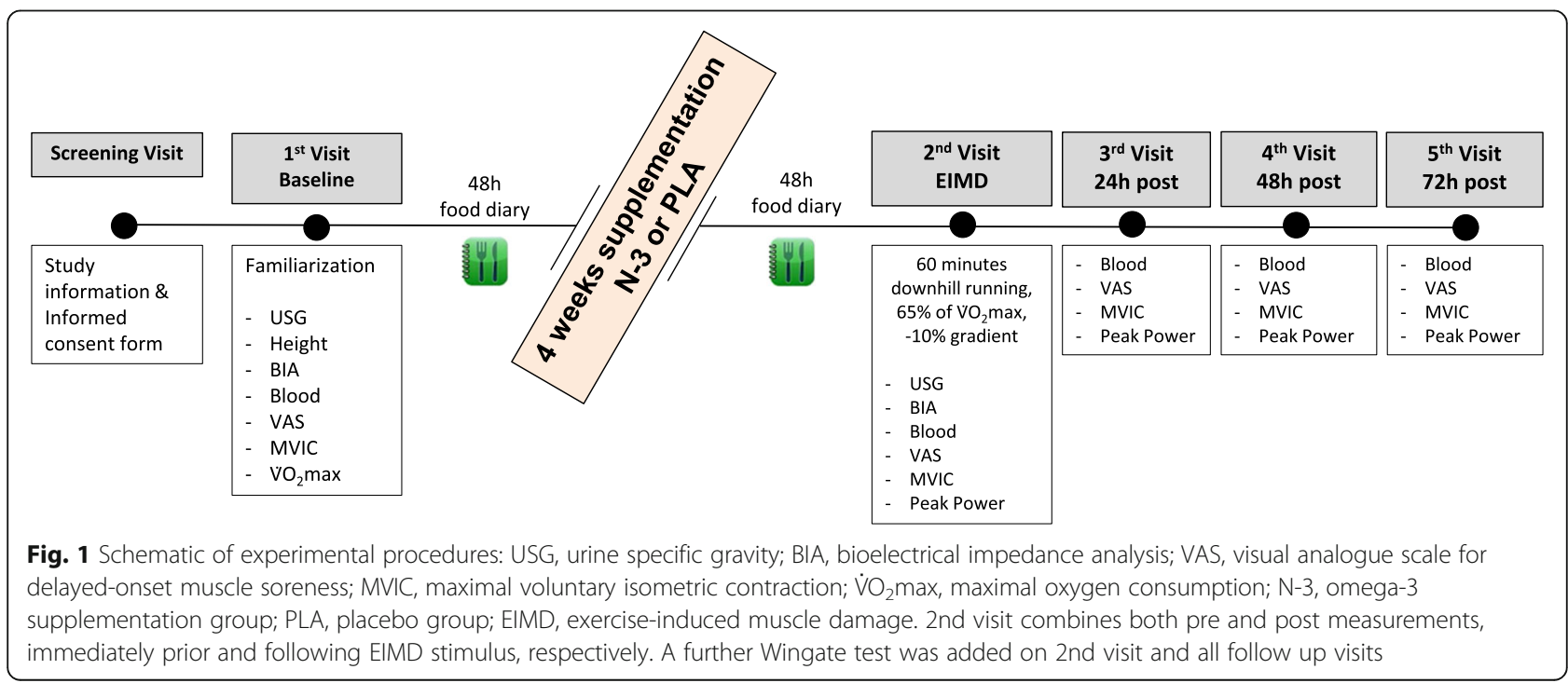


effects of $\mathrm{n}-3$ supplementation, such as unpleasant taste, heartburn, gastrointestinal discomfort and headache are usually mild [27], the amount of $n-3$ provided is in line with the nutritional recommendations as part of a normal diet and does not cause any harm or side effects. Daily supplementation of up to about $5 \mathrm{~g} /$ day of $\mathrm{n}-3$ PUFA in a long-term consumption is considered safe by the European Food Safety Authority (EFSA) [28]. Longer duration or high doses may affect immune function due to suppression of inflammatory response [29]. High doses also might increase bleeding time by reducing platelet aggregation [29]. The placebo group received $3 \times 600 \mathrm{mg}$ capsules per day of collagen (Troo Healthcare, Colchester, UK), consumed in a matching pattern. Participants were only given 1 week of capsules at a time. Initially written reminders were sent on a daily basis to ensure supplementation practices were maintained consistent throughout the day. Participants' compliance also verified by weekly written and oral reminders, counting remaining capsules at the end of each week and issuing of future weeks capsules took place. Further, participants were asked to guess what group they were at the conclusion of testing with 2 of 7 in placebo and 5 of 7 in N-3 group correctly guessing the supplementation group.

\section{Diet and activity control}

Participants were requested to maintain their usual diet and physical activity throughout the study. A 48-h food diary (including 1 day of the week and one weekend day) was provided to record all foods and drinks consumed prior to the supplementation period starting. Following 4 weeks of supplementation, participants completed a second 48-h food diary in the 2 days prior to the EIMD trial. Written and oral reminders were also provided on a regular basis to ensure diet and exercise practices were maintained consistent throughout the study.

Food diaries were analysed using Nutritics to quantify total energy intake, macronutrients (carbohydrates, protein, fatty acids), n-3 and n- 6 PUFA before and after the supplementation period. In addition, at the start of the supplementation period all participants were provided with a food list with foods low ( $<250 \mathrm{mg}$ per serving), moderate ( $250 \mathrm{mg}$ per serving) and high $(>500 \mathrm{mg}$ per serving) in omega-3 fatty acids to prevent increasing their omega-3 intake through diet. Cut-off points were used by USDA SR-21 (2008) [30].

\section{EIMD protocol}

Following a 3-min warm up, participants ran for $60 \mathrm{~min}$ at the individualized predetermined $V_{\text {test }}$ at $-10 \%$ gradient. Heart rate (HR) and rating of perceived exertion (RPE), Borg 6-20 scale [31] were recorded throughout the trial every $10 \mathrm{~min}$. A 60 -s sample expired of gases were collected at 20 and $40 \mathrm{~min}$ of trial and analysed by an on-line breath-by breath system for $\dot{\mathrm{V}}_{2}$ to ensure participants were running at $65 \% \dot{\mathrm{V}}_{2}$ max. Water was provided ad libitum every 15 min whilst running. Immediately after the muscle-damaging bout participants sat and a blood sample was collected (post-EIMD). Participants then rated their perceived muscle soreness, and MVIC and Wingate test were performed to assess participants' post-EIMD strength and power output, respectively.

\section{Venous plasma}

A total of $12 \mathrm{~mL}$ of venous blood was collected at each time point in two $6 \mathrm{~mL}$ vacutainer tubes $\left(\mathrm{K}_{2}\right.$ EDTA and lithium-heparin; BD, Oxford, UK). Haematocrit with capillary method using a micro-hematocrit reader (Hawksley \& Sons Ltd., Lancing, UK) and haemoglobin concentration using a photometer (Haemocue, Sheffield, UK) were both analysed immediately on heparinized whole blood in triplicate. Subsequently, concentration of plasma markers was adjusted for plasma volume changes with the method of Dill and Costill [32]. The remaining whole blood was spun (Hettich Universal 320 R, Germany) at $5000 \mathrm{rpm}$ for $10 \mathrm{~min}$ at $4{ }^{\circ} \mathrm{C}$, with plasma aliquoted and frozen at $-80^{\circ} \mathrm{C}$.

Circulating CK activity was measured using a clinical chemistry analyser (Werfen ILab Aries, Italy). CK activity was determined using kinetic spectrophotometry at $340 \mathrm{~nm}$ with a minimum detection limit of $3 \mathrm{U} / \mathrm{L}$, an undiluted linearity up to $900 \mathrm{U} / \mathrm{L}$. Coefficient of variation (CV) was within run $<1.2 \%$, total $<2.5 \%$. All samples and standards were analysed in duplicate.

Aliquots of plasma were analysed for plasma IL-6 and TNF- $\alpha$ concentration by enzyme-linked immunosorbent assay (ELISA) DuoSet kits and ancillary reagent packs (IL-6 DY206, TNF- $\alpha$, DY210, consumables DY008, RED Systems, USA) in duplicate, according to manufacturer's instruction. Plates were read at $450 \mathrm{~nm}$ and blanked to $590 \mathrm{~nm}$.

\section{Perceived muscle soreness}

Muscle soreness was self-rated by participants on a 10point-validated visual analogue scale (VAS) indicating on a line from 0 (no pain) to 10 (extreme pain) [33], during a wall squat with thighs parallel to the floor at $90^{\circ}$ degrees.

\section{Maximal voluntary isometric contraction}

MVIC was assessed on a dynamometer (Globus Kineo 7000 , Italy). The chair was adjusted so that the leg pad was placed on the lower part of the tibialis anterior and the pivot was located on the lateral epicondyle of the right leg. Maximal force was measured at an angle of 
$60^{\circ}$ leg extension. Peak force was determined by the average of four maximal isometric contractions lasting $3-5 \mathrm{~s}$. The contraction time was recorded by an experimenter.

\section{Peak power}

Participants performed a $10 \mathrm{~s}$ Wingate test on a cycle ergometer (Monark Ergomedic 894E, Sweden), fixed with an optical sensor (OptoSensor 2000 ${ }^{\mathrm{sm}}$, Sport Medicine Industries, USA) with the data obtained by the Monark Anaerobic Test Software. Participants cycled seated during the sprint protocol, with a resistance equal to $7.5 \%$ of their body weight. Participants were verbally encouraged throughout the test.

\section{Statistical analysis}

Normal distribution of all data was performed by the Shapiro-Wilk Test. Following Levene's test of equality of variance, baseline characteristics, dietary and hydration data were compared between groups using a two-tailed independent samples t-test. The examination of the effect of the $n-3$ supplementation on plasma CK activity, IL-6, TNF- $\alpha$ and DOMS was performed by nonparametric tests, as these variables did not follow normal distribution. Mann-Whitney $U$ test was performed to examine differences between N-3 and PLA group at each time point. A Freidman test was used to determine the main effect of time within-group and post hoc with Wilcoxon Signed Rank tests (using a Bonferroni adjusted alpha value) was run where a significant time was identified. MVIC and peak power data met all assumptions required for normality and were analysed using a two-way mixed between-within participant repeated measures analysis of variance (ANOVA). Bonferroni-adjust pairwise comparisons post hoc analysis was used where needed to examine within subject differences. Values were expressed as mean \pm SD for data from parametric tests and as median and interquartile range for data from non-parametric tests. Statistical significance was accepted as $p<0.05$. Effect size was calculated using methods proposed by Cohen [34], with effect sizes considered small (0.2), medium (0.5) or large (0.8). Statistical analyses were performed using SPSS 25 software (IBM SPSS, NY, USA). All figures were generated in GraphPad Prism (Version 8, GraphPad).

\section{Power calculation}

The sample size was estimated from a sample calculation $\left(G^{*}\right.$ Power 3.1) with an alpha level of 0.05 , a power $(1-\beta)$ of 0.80 and a medium effect size of 0.5 and suggests $n=12$ in total would be sufficient.

\section{Results}

\section{Descriptive characteristics}

The physical characteristics of participants completed EIMD are presented in Table 1. There was homogeneity in all characteristics of participants between groups.

Descriptive characteristics in dietary data at baseline (before supplementation) of participants from both groups are presented in Table 2. An independentsamples t-test was conducted to compare energy, macronutrients and n-6/n-3 ratio between groups, and MannWhitney $U$ test was performed to compare $n-3$ intake [N-3, Md = 7.57 (53.00); PLA, $\mathrm{Md}=7.43$ (52.00), $\mathrm{U}=$ 24.00, $\mathrm{Z}=-0.064]$ and $\mathrm{n}-6$ intake $[\mathrm{N}-3, \mathrm{Md}=8.21$ (57.50); PLA, Md=6.79 (47.50), $\mathrm{U}=19.50, \mathrm{Z}=-0.640$ ] between groups. There was no significant difference in food intake $(p>0.05)$ at baseline between N-3 and PLA group (Table 2).

Descriptive characteristics in dietary data after the supplementation period of participants from both groups are presented in Table 3. An independent-samples t-test was conducted to compare energy, macronutrients, n-3 and $n-6$ intake between groups. There was a significant difference in $\mathrm{n}-3$ intake with $\mathrm{N}-3$ group showing a higher n-3 intake post supplementation. However, there was no significant difference in any other food intake $(p>0.05)$ between groups (Table 3$)$.

In addition, an independent-samples t-test was conducted to compare participants' hydration status before EIMD between N-3 and PLA group. There was no significant difference in hydration status $[\mathrm{N}-3, n=7,(\mathrm{M}=$ $1.015, \mathrm{SD}=0.007)$ and PLA, $n=7, \quad(\mathrm{M}=1.012, \mathrm{SD}=$ $0.007) ; \mathrm{t}(12)=0.72, p>0.05$, two-tailed] between groups.

\section{Blood markers}

Friedman's ANOVA suggested an effect of time on CK activity for both PLA and N-3 groups (both $p<0.001$ ), with post hoc testing suggesting both groups were increased at 24, 48 and $72 \mathrm{~h}$ relative to baseline $(p<0.05$, $r=0.63$ indicating a medium effect size for all three time points; Fig. 2.

A). However, there was no significant difference between PLA and N-3 at any timepoint.

Mann-Whitney $U$ test was used to compare plasma IL-6 concentration between N-3 and PLA group. There was no significant difference between groups at any time point. Nevertheless, Friedman's ANOVA revealed plasma IL-6 did not change over time in N-3 group ( $p=$ $0.434)$ but did change in PLA group $(p=0.009)$. Post hoc testing suggested IL-6 was elevated in the PLA group at immediately post-EIMD relative to baseline $(p<0.05, r=0.61$ indicating a medium effect size) but no other time points (Fig. 2) 
Table 1 Physical characteristics of participants completed EIMD, independent sample t-test comparison between N-3 and Placebo group

\begin{tabular}{|c|c|c|c|c|}
\hline & $\begin{array}{l}\text { Total } \\
(n=14)\end{array}$ & $\begin{array}{l}\mathrm{N}-3 \\
(n=7)\end{array}$ & $\begin{array}{l}\text { PLA } \\
(n=7)\end{array}$ & $P$-value \\
\hline Age (years) & $25.07( \pm 4.05)$ & $25.57( \pm 4.18)$ & $24.57( \pm 4.24)$ & 0.66 \\
\hline Weight (kg) & $73.04( \pm 9.82)$ & $69.34( \pm 10.93)$ & $76.74( \pm 7.59)$ & 0.17 \\
\hline Height (cm) & $179.59( \pm 10.24)$ & $174.60( \pm 11.64)$ & $184.59( \pm 5.77)$ & 0.06 \\
\hline BMI $\left(\mathrm{kg} / \mathrm{m}^{2}\right)$ & $22.60( \pm 1.80)$ & $22.63( \pm 1.41)$ & $22.59( \pm 2.24)$ & 0.97 \\
\hline Body fat (\%) & $10.75( \pm 4.07)$ & $10.40( \pm 4.29)$ & $11.10( \pm 4.15)$ & 0.76 \\
\hline$\dot{\mathrm{V}}_{2} \max (\mathrm{ml} / \mathrm{kg} / \mathrm{min})$ & $62.43( \pm 11.77)$ & $65.11( \pm 11.08)$ & $59.74( \pm 12.66)$ & 0.41 \\
\hline
\end{tabular}

Values are expressed as mean \pm SD. N-3, omega-3 group; PLA placebo group, $B M I$ body mass index, $\dot{V} \mathrm{O}_{2}$ max maximal oxygen consumption

B). Mann-Whitney U test was performed to compare plasma TNF- $\alpha$ concentration between groups. Plasma TNF- $\alpha$ did not differ with time in either PLA $(p=0.274)$ or N-3 group ( $p=0.345$; Fig. $2 \mathrm{c}$ ).

\section{Functional measurements}

Mann-Whitney $U$ test was run to compare perceived muscle soreness between N-3 and PLA group at each time point. There was a statistically significant difference in DOMS between groups at $24 \mathrm{~h}$ post-EIMD, with PLA showing a higher muscle soreness compared to N-3 group $(p=0.034)$ with a medium effect size $(r=0.56)$. Friedman's test suggested DOMS significantly differed both within the N-3 and PLA group $(p<0.001)$. Pairwise comparisons suggested that N-3 group had elevated DOMS immediately post $(r=0.57)$ and at $24 \mathrm{~h}$ postEIMD $(r=0.59)$ relative to pre (all $p<0.05)$, whilst the PLA group maintained DOMS for longer, being elevated immediately post $(\mathrm{r}=0.64)$, and at both 24 and $48 \mathrm{~h}$ post-EIMD $(r=0.6)$ relative to pre (all $p<0.05$; Fig. 3a).

No group $\mathrm{x}$ time interaction was noted for MVIC (repeated measures ANOVA, $p=0.813$ ) or a main effect of group $(p=0.338)$. A significant main effect for time was observed for MVIC leg strength $\left(p=0.011, \eta_{\mathrm{p}}{ }^{2}=0.813\right)$. This result suggests a large effect size with MVIC suppressed relative to pre in both $\mathrm{N}-3$ and PLA groups $(\mathrm{N}-$
$3=29.6( \pm 8.8) \mathrm{kg}$ vs $20.8( \pm 10.6) \mathrm{kg}, \mathrm{PLA}=36.5( \pm 10.2)$ $\mathrm{kg}$ vs $27.1( \pm 7.4) \mathrm{kg}$; both $p<0.05)$ immediately postEIMD, but no other time points (Fig. $3 \mathrm{~b}$ ).

No group $\mathrm{x}$ time interaction was noted for peak power (repeated measures ANOVA, $p=0.514$ ) or a main effect of group $(p=0.310)$. A significant main effect for time was observed for peak power $\left(p=0.014, \eta_{\mathrm{p}}{ }^{2}=0.841\right)$. This result suggests a large effect size with post hoc testing suggesting no change in peak power following EIMD in N-3 group, but a suppression in peak power in PLA group at $24 \mathrm{~h}$ relative to pre [pre-EIMD $=825.6( \pm 90.7)$, $24 \mathrm{~h}=763.0( \pm 103.1) \mathrm{W}, p<0.05$ ] (Fig. 3c).

\section{Discussion}

Results presented here suggest that 4 weeks supplementation with $3 \mathrm{~g} /$ day of $\mathrm{n}-3$ PUFA offsets the EIMD induced pain response following a single bout of high intensity exercise. Whilst a decreased peak power output in PLA group at $24 \mathrm{~h}$ following EIMD and a blunted time response in pro-inflammatory marker IL-6 was witnessed in the N-3 group immediately post-EIMD, no between group differences were found. Although findings suggest decreased MVIC and increased plasma CK following EIMD, no difference was observed between groups, overall suggesting minimal positive gain in exercise performance with n-3 supplementation.

Table 2 Dietary data at baseline between N-3 and PLA groups

\begin{tabular}{|c|c|c|c|c|}
\hline & $\begin{array}{l}\text { Total } \\
(n=14)\end{array}$ & $\begin{array}{l}\mathrm{N}-3 \\
(n=7)\end{array}$ & $\begin{array}{l}\text { PLA } \\
(n=7)\end{array}$ & $P$-value \\
\hline Energy (Kcals) & $2580.64( \pm 789.39)$ & $2664.71( \pm 414.35)$ & $2496.57( \pm 1077.93)$ & 0.707 \\
\hline $\mathrm{CHO}(\mathrm{g})$ & $296.64( \pm 83.62)$ & $319.71( \pm 59.15)$ & $273.57( \pm 102.02)$ & 0.321 \\
\hline Protein (g) & $109.11( \pm 30.21)$ & $108.64( \pm 23.20)$ & $109.57( \pm 37.94)$ & 0.957 \\
\hline Fat (g) & $94.99( \pm 46.12)$ & $89.48( \pm 28.06)$ & $100.49( \pm 61.23)$ & 0.673 \\
\hline$n-3(g)^{a}$ & $1.56( \pm 1.43)$ & $1.41( \pm 1.20)$ & $1.70( \pm 1.72)$ & 0.949 \\
\hline$n-6(g)^{a}$ & $9.47( \pm 7.42)$ & $9.85( \pm 6.59)$ & $9.09( \pm 8.69)$ & 0.522 \\
\hline$n-6 / n-3$ ratio & $8.20( \pm 4.91)$ & $9.18( \pm 4.81)$ & $7.21( \pm 5.18)$ & 0.476 \\
\hline
\end{tabular}

Values are expressed as mean \pm SD. $N-3$ omega-3 group, PLA placebo, $C H O$ carbohydrates, $n-3$ omega-3 fatty acids, $n-6$ omega- 6 fatty acids. ${ }^{\text {indicate }}$ MannWhitney $U$ test 
Table 3 Dietary data post supplementation (+ $3 \mathrm{~g}$ of $\mathrm{n}-3$ supplementation) of participants completed EIMD, independent sample ttest comparison between $\mathrm{N}-3$ and Placebo group

\begin{tabular}{|c|c|c|c|c|}
\hline & $\begin{array}{l}\text { Total } \\
(n=14)\end{array}$ & $\begin{array}{l}\mathrm{N}-3 \\
(n=7)\end{array}$ & $\begin{array}{l}\text { PLA } \\
(n=7)\end{array}$ & $P$-value \\
\hline Energy (Kcals) & $2574.57( \pm 928.64)$ & $2653.43( \pm 794.99)$ & $2495.71( \pm 1105.43)$ & 0.765 \\
\hline $\mathrm{CHO}(\mathrm{g})$ & $305.64( \pm 134.93)$ & $293.50( \pm 68.18)$ & $317.78( \pm 185.62)$ & 0.751 \\
\hline Protein (g) & $109.79( \pm 32.36)$ & $104.06( \pm 26.08)$ & $115.52( \pm 38.89)$ & 0.529 \\
\hline Fat (g) & $92.84( \pm 37.37)$ & $104.33( \pm 38.87)$ & $81.35( \pm 34.74)$ & 0.266 \\
\hline$n-3(g)$ & $2.82( \pm 1.92)$ & $3.87( \pm 1.90)$ & $1.78( \pm 1.34)$ & $0.036^{*}$ \\
\hline$n-6(g)$ & $8.47( \pm 5.41)$ & $8.65( \pm 5.83)$ & $8.29( \pm 5.42)$ & 0.906 \\
\hline n-6/n-3 ratio & $4.65( \pm 3.80)$ & $3.19( \pm 3.18)$ & $6.12( \pm 4.03)$ & 0.157 \\
\hline
\end{tabular}

"Significant level, $p<.05$; Values are expressed as mean \pm SD. $N-3$ omega-3 group, PLA placebo, CHO carbohydrates, $n-3$ omega-3 fatty acids, $n-6$ omega-6 fatty acids

\section{Blood markers}

Our data show a significant increase of CK activity following EIMD before returning to baseline in both groups, mirroring those previously reported [9, 35]. Conversely, Bloomer et al. [20] reported no significantly increased CK activity following eccentric exercise. This finding is in agreement with the findings by Atashak et al. [19], another study [36] with a similar exercise protocol, following a 40 min downhill running, and Tsuchiya et al. [23] after an eccentric protocol with a similar dose but longer supplementation period (8 weeks).

The data presented here showed a large degree of variability in circulating CK (from 65 to $4939 \mathrm{U} / \mathrm{L}$ ) which is in accordance with previous research [37]. Hence, CK alone might not be an accurate reflection of the degree of muscle damage following eccentric exercise [38] due to large inter-individual variability in response with a range from 236 to 25,244 IU/L [1]. Therefore, the results of the efficacy of the $n-3$ supplementation on indirect muscle damage biomarkers, such as CK, following maximal exercise performance may be inconsistent due to variability alone, and such markers should not be considered in isolation.

Plasma IL-6 concentration peaked immediately postEIMD for the PLA group. This peak of plasma IL-6 after exercise is well documented in the literature [39-41]. However, there was no significant difference in plasma IL-6 concentration between N-3 and PLA group. This finding is in accordance with the findings by Tarbinian et al. [41], who found no significant difference between groups in plasma IL- 6 concentration immediately postexercise.

In a manner similar to plasma IL-6, there were no differences in post-EIMD plasma TNF- $\alpha$ concentration between N-3 and PLA groups. There is conflicting evidence about the behaviour of TNF- $\alpha$ response after muscle-damaging exercise. Toft et al. [42] have shown that plasma TNF- $\alpha$ was elevated after prolonged exercise, others did not observe any change [43] and others recorded a decrease in the TNF- $\alpha$ [44]. In the study by Lenn et al. [45], TNF- $\alpha$ was not significantly increased, which is a similar result with the present study, where no significant increase was demonstrated in plasma TNF- $\alpha$ concentration. This could be due to a feedback mechanism, that IL-6 inhibits TNF- $\alpha$ [43]. Thus, it may be that plasma TNF- $\alpha$ is not an optimal marker to quantify EIMD-induced inflammation.

\section{Functional measurements}

We report a significant change in VAS pain score following EIMD in both groups, further evidence that the exercise protocol used caused significant muscle damage. More specifically, participants' pain perception peaked immediately post-EIMD and remained elevated at 24-48 $\mathrm{h}$ post-EIMD, which is consistent with other findings $[1,46]$. Our study demonstrated a significant difference in perceived muscle soreness between groups at $24 \mathrm{~h}$ post-EIMD, suggesting $\mathrm{N}-3$ may have experienced less pain compared to PLA group at this point. Previous studies [17, 18, 47] also found significant differences in DOMS between groups following EIMD, with the fish oil group having reduced muscle soreness. On the contrary, Jakeman et al. [17] an acute dose of n-3 PUFA immediately after a muscle-damaging exercise, demonstrated similar muscle soreness between groups. The absence of effect on DOMS might be due to the acute supplementation dose following exercise and is insufficient to change muscle phospholipid content $[48,49]$ relative to the 4 weeks of supplementation used here.

Subsequent exercise performance is significantly affected by EIMD and its symptoms [2]. The loss of muscle force is considered the most valid indirect measurement of muscle damage [50]. As expected, and when observing a large effect size, the leg strength significantly decreased immediately post-EIMD in both groups compared with pre-EIMD values. However, there were no significant differences in MVIC between groups nor was any interaction effect observed, suggesting that levels of muscle damage were 


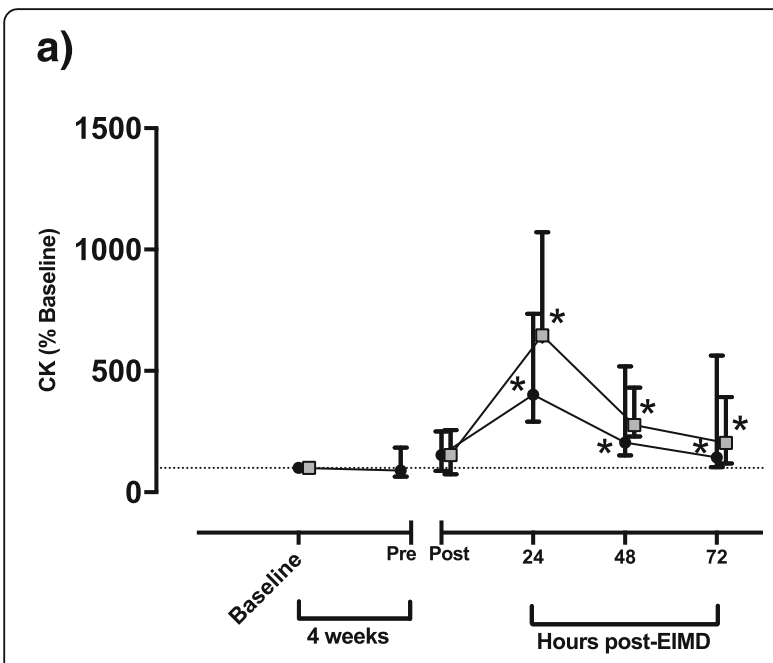

b)
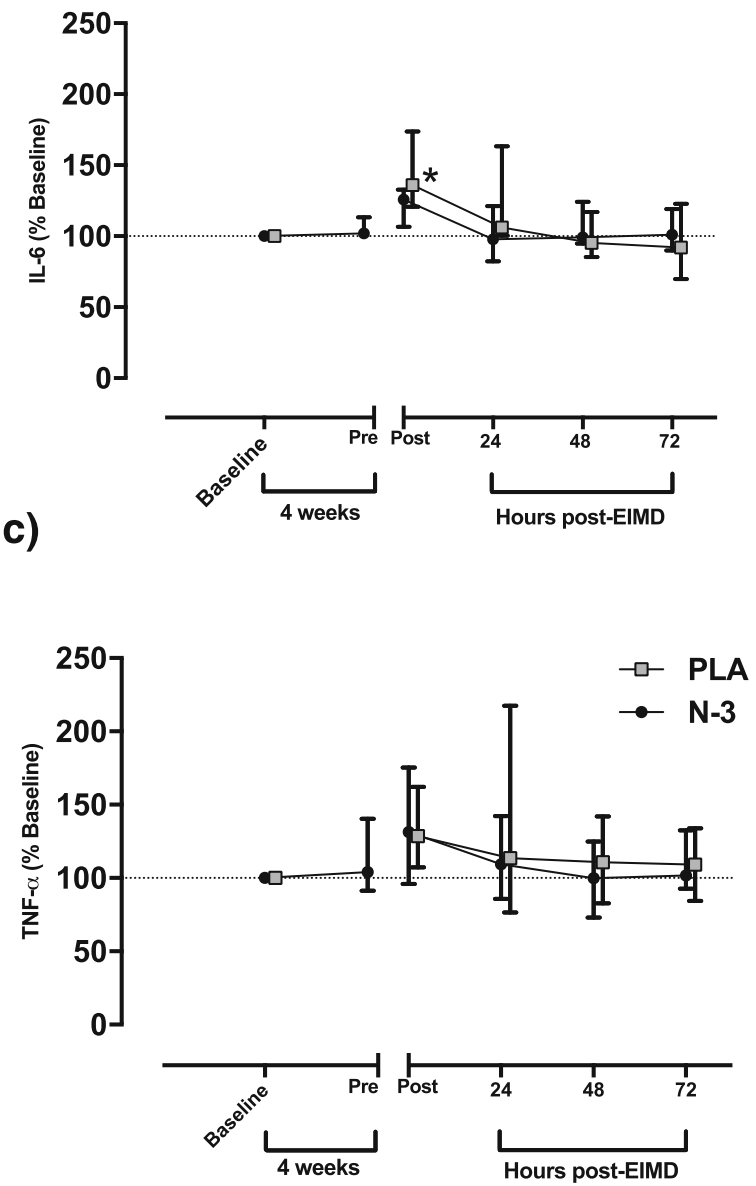

Fig. 2 Plasma markers of muscle damage and inflammation as a function of time. a CK (\% change from baseline), $\mathbf{b}$ IL-6 (\% change from baseline), and c TNF-a (\% change from baseline). Data shown as medians, error bars indicate interquartile range. Dashed horizontal line indicates 100\% (baseline). ${ }^{*}$ indicates significant difference from baseline. Time matched data points offset horizontally to enhance clarity

a)

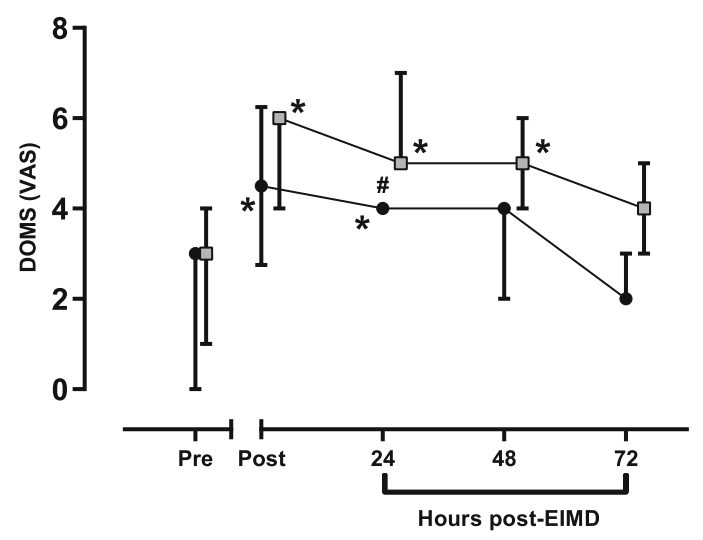

b)
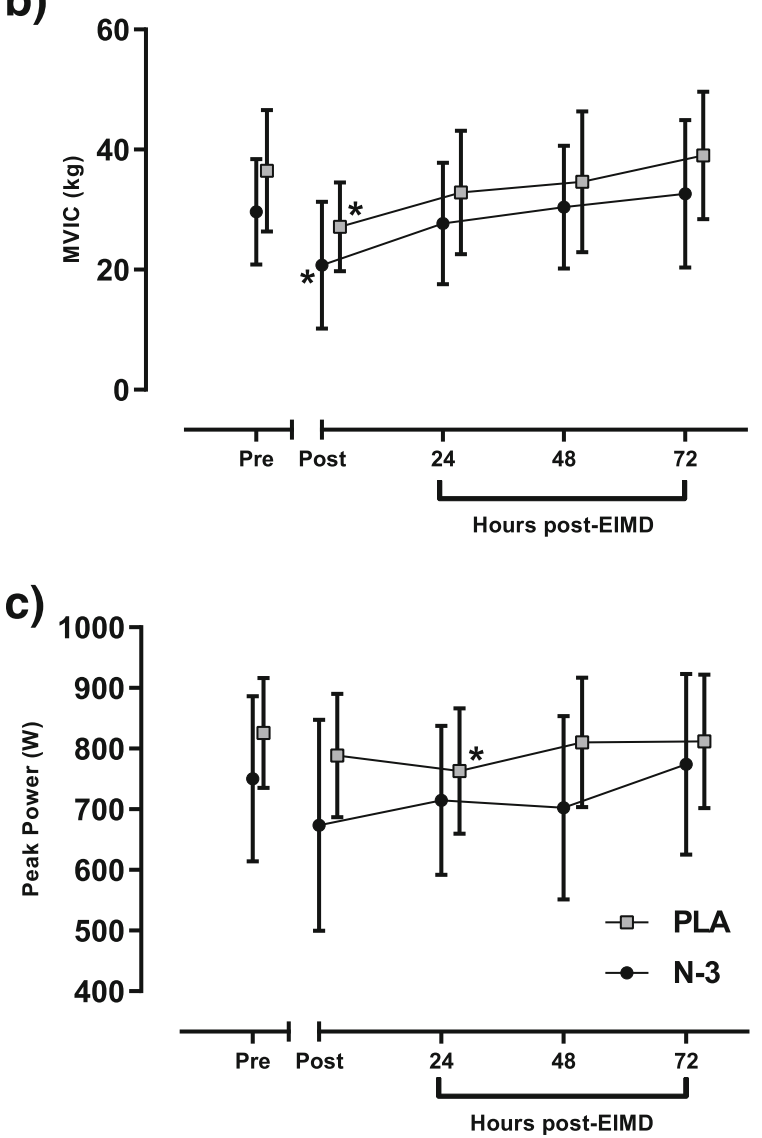

Fig. 3 Muscle functional measures prior (pre) and following (post 72 h) EIMD. a DOMS, data indicates median, error bars interquartile range. Both b MVIC $(\mathrm{Kg})$, and c peak power $(\mathrm{W})$ data indicate means, error bars standard deviation. * indicates significant difference from Pre timepoint, \# indicates difference between groups at timepoint indicated. Time matched data points offset horizontally to enhance clarity 
unchanged by $\mathrm{n}-3$ consumption. These findings match both those of Gravina et al. [51], who reported no impact of 4 weeks of n-3 supplementation on leg strength, despite a higher dose (equivalent of $0.1 \mathrm{~g} / \mathrm{kg} /$ day of supplement in capsules, $1000 \mathrm{mg}$ n-3 PUFA per capsule, with a mean intake of $7 \pm 2$ capsules per day) and of Gray et al. [20] who showed no group effect, with a longer duration ( 6 weeks) of $\mathrm{n}-3$ supplementation. In addition, a very recent study by Ramos-Campo et al. [52], examining muscle damage after eccentric exercise, found no significant difference in strength deficit between the supplementation and placebo group following a 10-week n-3 supplementation. Therefore, the implications of the findings from the previous studies and ours are that $n-3$ supplementation does not have significant positive effects on muscle strength recovery.

As a secondary measure of muscle function, we examined cycling peak power, with no significant difference between groups. However, the PLA group demonstrated a significant suppression in peak power at $24 \mathrm{~h}$ following EIMD relative to pre, while there was no change in $\mathrm{N}-3$ group relative to pre-EIMD. Decreased cycling peak power output 24h following EIMD is in line with previous research [53]. The potential for preservation of voluntary peak power output will be of interest to athletes where repeated maximal powerful performance is required, which is reinforced by differences in perceived pain at this timepoint.

In addition, we examined participants' hydration status before EIMD to ensure they began exercise euhydrated, to prevent hydration being a cofounding factor for exercise performance. We also assessed n-3 intake using a 48-h food diary at pre- and post-supplementation period. No difference in $n-3$ intake was noted between groups prior to supplementation. As it would be expected, there was an increase in $\mathrm{n}-3$ intake in the $\mathrm{N}-3$ group relative to the PLA group after supplementation.

\section{Limitations, recommendations and future directions}

Some potential limitations of the present study should be acknowledged. Low statistical power due to the modest sample size played a role in limiting the significance of the statistical comparisons conducted. The strict inclusion criteria as well as the downhill running task performance made recruitment for participants difficult. Additional blood biomarkers, such as myoglobin and C-reactive protein, may also provide further information in future studies on muscle damage. A clearer picture on change in muscle function could involve examining $n-3$ supplementation and muscle damage considering multiple functional measurements, such as MVC torque at multiple joint angles, ROM, limb swelling and/or jump height. Measurements of muscle function should be used in combination with indirect plasma markers to provide more reliable evidence in assessing the magnitude of muscle damage. Ideally, directly measuring muscle damage from muscle biopsies would be optimal, albeit highly invasive. Additionally, future studies may consider taking blood samples at additional acute time points, such as 1, 3, 6 or $12 \mathrm{~h}$ after the muscle-damaging exercise. By doing so, we might have observed an acute inflammatory response difference between groups, as has been observed elsewhere [17]. Participants' diets were not explicitly controlled during the 4-week loading period, however, a 48-h food diary was recorded immediately before supplementation period and repeated $48 \mathrm{~h}$ before supplementation finished (immediately before EIMD); results of which suggested participants did not change their habitual macronutrient or total caloric intake. Future studies may consider a method to control participants' food intake, such as providing pre-packaged meals or recording complete food diaries throughout both supplementation period and recovery phase. However, this would incur both significant cost and require participants to have a greater commitment to these methods. In an attempt to isolate the effect of $n-3$ supplementation, collagen was chosen as the placebo in this study to avoid manipulation of $n-6 / n-$ 3 ratio. There is no evidence in the literature that collagen has a pro or anti-inflammatory effect, and therefore, it would not oppose the action of $n-3$ supplementation. Whereas, other reports have utilized corn oil as a placebo control which is high in $\mathrm{n}-6$, and thus may not represent a true placebo $[11,54]$.

\section{Conclusion}

Overall, these findings support the hypothesis that 4 weeks of $3 \mathrm{~g} /$ day $\mathrm{n}-3$ supplementation may attenuate minor aspects of EIMD, as observed in DOMS and peak power. Typically, no significant differences were noted between groups, however, it was observed a blunted inflammatory response immediately after eccentric exercise and a decreased CK activity at $24 \mathrm{~h}$ following muscle-damaging exercise in N-3 group. There were also no significant differences in leg strength between groups indicating that n-3 supplementation will have limited impact on muscle function and subsequent performance. Whilst not improving performance, these findings may have relevance to soreness-associated exercise avoidance.

\section{Supplementary Information}

The online version contains supplementary material available at https://doi. org/10.1186/s12970-020-00405-1.

\section{Additional file 1 \\ Additional file 2. \\ Additional file 3.}

\section{Abbreviations}

ANOVA: Analysis of variance; AA: Arachidonic acid; BIA: Bioelectrical impedance analysis; BMI: Body mass index; CK: Creatine kinase; CV: Coefficient of variation; DHA: Docosahexaenoic acid; DOMS: Delayedonset muscle soreness; EIMD: Exercise-induced muscle damage; ELISA: Enzyme-linked immunosorbent assay; EPA: Eicosapentaenoic acid; Hb: Haemoglobin; Hct: Haematocrit; HR: Heart rate; IL-1ra: Interleukin-1 
receptor; IL-6: Interleukin-6; IL-10: Interleukin-10; MVIC: Maximal voluntary isometric contraction; n-3 PUFA: Omega-3 polyunsaturated fatty acids; N3: Omega-3 interventional group; PAR-Q: Physical activity readiness questionnaire; PLA: Placebo group; Post-EIMD: Following exercise-induced muscle damage; Pre-EIMD: Before exercise-induced muscle damage; ROM: Range of motion; ROS: Reactive oxygen species; RPE: Rating of perceived exertion; RPM: Revolutions per minute; SD: Standard deviation; TNF-a: Tumor necrosis factor-alpha; USG: Urine specific gravity; VAS: Visual analogue scale; $\dot{V}_{2}$ : Absolute oxygen consumption; $\dot{\mathrm{V}}_{2}$ max: Maximal oxygen consumption; Vtest: Downhill running speed; WURSS: Wisconsin upper respiratory symptom survey

\section{Acknowledgments}

The authors would like to thank all the volunteers participated in this study. Thanks also to Kate Edwards, Isabela Ramos, John Sampson and Marlene Ferreira for assistance with data collection, Isabella Cooper for theoretical discussions and Helen Lloyd for technical support.

\section{Authors' contributions}

This work was completed in the Translational Physiology Research Group, University of Westminster. YK for formal analysis and investigation, YK and CF for data curation, YK and BE for visualization, YK and AD for methodology, YK and $\mathrm{BE}$ for project administration, $\mathrm{BE}$ and $\mathrm{AD}$ for funding acquisition, $\mathrm{YK}$ for writing - original draft manuscript, YK, CW, CF, AD and BE for writing review \& editing. All authors read and approved the final manuscript. BE is the corresponding author for this paper.

\section{Funding}

BE is supported by the Quintin Hogg Charitable Trust and AD by internal University of Westminster funding.

\section{Availability of data and materials}

The datasets generated and analysed during the current study are available as supplementary material from the corresponding author on reasonable request.

\section{Ethics approval and consent to participate}

All participants signed a written informed consent. The study was performed in accordance with the guidelines in the Declaration of Helsinki and was approved by the College of Liberal of Arts and Sciences Research Ethics Committee, University of Westminster (ETH1617-0182).

\section{Consent for publication}

Not applicable.

\section{Competing interests}

The Kineo Isokinetic dynamometer utilized was provided to the University of Westminster without cost for testing and development by Globus Italia.

\section{Author details}

${ }^{1}$ Translational Physiology Research Group, School of Life Sciences, College of Liberal Arts and Sciences, University of Westminster, London W1W 6UW, UK. ${ }^{2}$ School of Sport, Rehabilitation and Exercise Sciences, University of Essex, Essex, UK.

Received: 16 June 2020 Accepted: 26 December 2020

Published online: 13 January 2021

\section{References}

1. Clarkson PM, Hubal MJ. Exercise-induced muscle damage in humans. Am J Phys Med Rehabil. 2002;81:52-69 [PubMed].

2. Owens DL, Twist C, Cobley JN, Howatson G, Close GL. Exercise-induced muscle damage: What is it, what causes it and what are the nutritional solutions? Eur J Sport Sci. 2019;19(1):71-85 [PubMed].

3. Paulsen G, Mikkelsen UR, Raastad T, Peake JM. Leucocytes, cytokines and satellite cells: what role do they play in muscle damage and regeneration following eccentric exercise? Exerc Immunol Rev. 2012;18:42-97 [PubMed].

4. Hyldahl RD, Hubal MJ. Lengthening our perspective: Morphological, cellular and molecular responses to eccentric exercise. Muscle Nerve. 2014;49:15570 [PubMed].
5. Ives JS, Bloom S, Matias A, Morrow N, Martins N, Roh Y, Ebenstein D, O'Brien G, Escudero D, Brito K, Glickman L, Connelly S, Arciero PJ. Effects of a combined protein and antioxidant supplement on recovery of muscle function and soreness following eccentric exercise. J Int Soc Sports Nutr. 2017;14:21 [PubMed].

6. Wan JJ, Qin Z, Wang PY, Sun Y, Liu X. Muscle fatigue: General understanding and treatment. Exp Mol Med. 2017;49:e384 [PubMed].

7. Peak JM, Neubauer O, Della Gatta PA, Nosaka K. Muscle damage and inflammation during recovery from exercise. J Appl Physiol. 2017;122:559-70 [PubMed].

8. Duque GA, Descoteaux A. Macrophage cytokines: involvement in immunity and infectious diseases. Front in Immunol. 2014;5:1-12 [PubMed].

9. Baird MF, Graham SM, Baker GJ, Bickerstaff GF. Creatine kinase and exercise related muscle damage implications for muscle performance and recovery. J Nutr Metabol. 2012;2012:1-13 [PubMed].

10. Calder PC. Omega-3 fatty acids and inflammatory processes: from molecules to man. Biochem Soc Trans. 2017:45(5):1105-15 [PubMed].

11. Philpott JD, Witard OC, Galloway SDR. Applications of omega-3 polyunsaturated fatty acid supplementation for sport performance. Res Sports Med. 2019;27(2):219-37 [PubMed].

12. Mickleborough TD. Omega-3 polyunsaturated fatty acids in physical performance optimization. Int J Sports Nutr Exerc Metab. 2013;23:83-96 [PubMed].

13. Shei RJ, Lindley MR, Mickleborough TD. Omega-3 polyunsaturated fatty acids in the optimization of physical performance. Mil Med. 2014;179:14456 [PubMed].

14. Walser B, Giordano RM, Stebbins CL. Supplementation with omega-3 polyunsaturated fatty acids augments brachial artery dilation and blood flow during forearm contraction. Eur J Appl Physiol. 2006;97:347-54 [PubMed].

15. Tartibian B, Maleki BH, Abbasi A. The effects of ingestion of omega-3 fatty acids on perceived pain and external symptoms of delayed onset muscle soreness in untrained men. Clin J Sport Med. 2009;19:115-9 [PubMed].

16. Jouris KB, McDaniel JL, Weiss EP. The effect of omega-3 fatty acid supplementation on the inflammatory response to eccentric strength exercise. J Sports Sci Med. 2011;10:432-8 [PubMed].

17. Jakeman JR, Lambrick DM, Wooley B, Babraj JA, Faulkner JA. Effect of an acute dose of omega-3 fish oil following exercise-induced muscle damage. Eur J Appl Physiol. 2017;117:575-82 [PubMed].

18. Lv Z, Zhang J, Zhu W. Omega-3 polyunsaturated fatty acid supplementation for reducing muscle soreness after eccentric exercise: A systematic review and meta-analysis of randomized controlled trials. Biomed Res Int. 2020; 2020:1-16 [PubMed].

19. Atashak S, Sharafi H, Azarbayjani MA, Stannard SR, Goli MA, Haghighi MM. Effect of omega-3 supplementation on the blood levels of oxidative stress, muscle damage and inflammation markers after acute resistance exercise in young athletes. Kinesiology. 2013;45:22-9 [Semantic Scholar].

20. Bloomer RJ, Larson DE, Fisher-Wellman KH, Galpin AJ, Schilling BK. Effect of Eicosapentaenoic and docosahexaenoic acid on resting and exerciseinduced inflammatory and oxidative stress biomarkers: A randomized, placebo controlled, cross-over study. Lipids Health Dis. 2009;8:36 [PubMed].

21. DiLorenzo FM, Drager CJ, Rankin JW. Docosahexaenoic acid affects markers of inflammation and muscle damage after eccentric exercise. J Strength Cond Res. 2014;28:2768-74 [PubMed].

22. Gray P, Chappell A, Jenkinson AM, Thies F, Gray SR. Fish oil supplementation reduces markers of oxidative stress but not muscle soreness after eccentric exercise. Int J Sports Nutr Exerc Metab. 2014;24:206-14 [PubMed].

23. Tsuchiya Y, Yanagimoto K, Nakazato K, Hayamizu K, Ochi E. Eicosapentaenoic and docosahexaenoic acids-rich fish oil supplementation attenuates strength loss and limited joint range of motion after eccentric contractions: A randomized, double-blind, placebo-controlled, parallel-group trial. Eur J Appl Physiol. 2016;1 16:1179-88 [PubMed].

24. Barrett B, Brown RL, Mundt MP, Thomas GR, Barlow SK, Highstrom AD, Bahrainian M. Validation of a short form Wisconsin upper respiratory symptom survey (WURSS-21). Health Qual Life Outcomes. 2009;7:76 [PubMed].

25. Dolci A, Fortes MB, Walker FS, Haq A, Riddle T, Walsh NP. Repeated muscle damage blunts the increase in heat strain during subsequent exercise heat stress. Eur J App Physiol. 2015;115:1577-88 [PubMed].

26. Armstrong LE, Pumerantz AC, Fiala KA, Roti MW, Kavouras SA, Casa DJ, Maresh CM. Human hydration indices: acute and longitudinal reference values. Int J Sport Nutr Exerc Metab. 2010;20:145-53 [PubMed]. 
27. Mazereeuw G, Lanctot KL, Chau SA, Swardfager W, Herrmann N. Effects of omega-3 fatty acids on cognitive performance: a meta-analysis. Neurobiol Aging. 2012;33:e17-29 [PubMed].

28. EFSA Panel on Dietetic Products. Scientific opinion on the tolerable upper intake level of eicosapentaenoic acid (EPA), docosahexaenoic acid (DHA) and docosapentaenoic acid (DPA). EFSA J. 2012;10:2815 [EFSA].

29. Institute of Medicine, Food and Nutrition Board. Dietary reference intakes for energy, carbohydrate, fiber, fat, fatty acids, cholesterol, protein, and amino acids (macronutrients). Washington, DC: National Academy Press; 2005. [The National Academies].

30. U.S. Department of Agriculture, Agricultural Research Service. USDA National Nutrient Database for Standard Reference, Release 21. In: Methods and Application of Food Composition Laboratory; 2008. Available from: [USDA].

31. Borg GA. Psychophysical bases of perceived exertion. Med Sci Sports Exerc. 1982;14:377-81 [PubMed].

32. Matomaki $\mathrm{P}$, Kainulainen $\mathrm{H}$, Kyrolainen $\mathrm{H}$. Corrected whole blood biomarkers - the equation of Dill and Costill revisited. Physiol Rep. 2018;6: e13749 [PubMed].

33. Carlsson AM. Assessment of chronic pain. I. Aspects of the reliability and validity of the visual analogue scale. Pain. 1983;16:87-101 [PubMed].

34. Cohen JW. Statistical power analysis for the behavioral sciences. 2nd ed. Hillsdale: Lawrence Erlbaum Associates; 1988.

35. Hughes JD, Denton K, Lloyd RS, Oliver JL, De Ste Croix M. The impact of soccer match play on the muscle damage response in youth female athletes. Int J Sports Med. 2018;39:343-8 [PubMed].

36. Park KS, Lee MG. Effects of unaccustomed downhill running on muscle damage, oxidative stress and leukocyte apoptosis. J Exerc Nutr Biochem. 2015;19:55-63 [PubMed]

37. Kim J, Lee J. The relationship of creatine kinase variability with body composition and muscle damage markers following eccentric muscle contractions. J Ex Nutr Biochem. 2015;19:123 [PubMed].

38. Magal M, Dumke C, Urbiztondo Z, Cavill M, Triplett N, Quindry J, Mcbride J, Epstein Y. Relationship between serum creatine kinase activity following exercise-induced muscle damage and muscle fibre composition. J Sports Sci. 2010;28:257-66 [PubMed].

39. Pedersen BK, Hoffman-Goetz L. Exercise and the immune system: regulation, integration, and adaptation. Physiol Rev. 2000;80:1055-81 [PubMed].

40. Smith LL, McKune AJ, Semple SJ, Sibanda E, Steel H, Anderson R. Changes in serum cytokines after repeated bouts of downhill running. Appl Physiol Nutr Metab. 2007;32:233-40 [PubMed].

41. Tartibian B, Maleki BH, Abbasi A. Omega-3 fatty acids supplementation attenuates inflammatory markers after eccentric exercise in untrained men. Clin J Sports Med. 2011;21:131-7 [PubMed].

42. Toft AD, Thorn M, Ostrowski K, Asp S, Moller K, Iversen S, Hermann C, Sondergaard SR, Pedersen BK. N-3 polyunsaturated fatty acids do not affect cytokine response to strenuous exercise. J App Physiol. 2000;89:2401-6 [PubMed].

43. Philippou A, Maridaki M, Psarros C, Koutsilieris M. Systemic responses of inflammation-related factors following eccentric exercise in humans. Am J Sports Sci. 2018;6:32-7 [SciencePG].

44. Hirose L, Nosaka K, Newton M, Laveder A, Kano M, Peake J, Suzuki K Changes in inflammatory mediators following eccentric exercise of the elbow flexors. Exerc Immunol Rev. 2004;10:75-90 [PubMed].

45. Lenn J, Uhl T, Mattacola C, Boissonneault G, Yates J, Ibrahim W, Bruckner G. The effects of fish oil and isoflavones on delayed onset muscle soreness. Med. Sci Sports Exerc. 2002;34:1605-13 [PubMed].

46. Chen TC, Lin MJ, Chen HL, Lai JH, Yu HI, Nosaka K. Muscle damage protective effect by two maximal isometric contractions on maximal eccentric exercise of the elbow flexors of the contralateral arm. Scand J Med Sci Sports. 2018;28:1354-60 [PubMed].

47. Ochi E, Tsuchiya Y, Yanagimoto K. Effect of eicosapentaenoic acids-rich fish oil supplement on motor nerve function after eccentric contractions. J of the Intern Soc of Sports Nutr. 2017;14:23 [PubMed].

48. McGlory C, Galloway SDR, Hamilton DL, McClintock C, Breen L, Dick JR, Bell $J G$, Tipton KD. Temporal changes in human skeletal muscle and blood lipid composition with fish oil supplementation. Prostaglandins Leukot Essent Fatty Acids. 2014;90(6):199-206 [PubMed].

49. Gerling CJ, Mukai K, Chabowski A, Heigenhauser GJF, Holloway GP, Spriet $L L$, Jannas-Vela S. Incorporation of omega-3 fatty acids into human skeletal muscle sarcolemmal and mitochondrial membranes following 12 weeks of fish oil supplementation. Front Physiol. 2019;10:348 [PubMed].
50. Damas F, Nosaka K, Libardi CA, Chen TC, Ugrinowitsch C. Susceptibility to exercise-induced muscle damage: a cluster analysis with a large sample. Int J Sports Med. 2016;37:633-40 [PubMed].

51. Gravina L, Brown FF, Alexander L, Dick J, Bell G, Witard OC, Galloway SDR. $\mathrm{N}-3$ fatty acid supplementation during 4 weeks of training leads to improved anaerobic endurance capacity, but not maximal strength, speed or power in soccer players. Int J Sport Nutr Exerc Metabol. 2017;27:305-13 [PubMed].

52. Ramos-Campo DJ, Avila-Gandia V, Lopez-Roman FJ, Minarro J, Contreras C, Soto-Mendez F, Domingo Pedrol JC, Luque-Rubia AJ. Supplementation of re-esterified docosahexaenoic and eicosapentaenoic acids reduce inflammatory and muscle damage markers after exercise in endurance athletes: a randomized, controlled crossover trial. Nutrients. 2020;12:719 [PubMed].

53. Twist C, Eston R. The effects of exercise-induced muscle damage on maximal intensity intermittent exercise performance. Eur J App Physiol. 2005;94:652-8 [PubMed].

54. Jeromson S, Gallagher IJ, Galloway SDR, Hamilton DL. Omega-3 fatty acids and skeletal muscle health. Mar Drugs. 2015;13:6977-7004 [PubMed].

\section{Publisher's Note}

Springer Nature remains neutral with regard to jurisdictional claims in published maps and institutional affiliations.
Ready to submit your research? Choose BMC and benefit from:

- fast, convenient online submission

- thorough peer review by experienced researchers in your field

- rapid publication on acceptance

- support for research data, including large and complex data types

- gold Open Access which fosters wider collaboration and increased citations

- maximum visibility for your research: over $100 \mathrm{M}$ website views per year

At BMC, research is always in progress.

Learn more biomedcentral.com/submissions 